\title{
OSCILLATION CRITERIA FOR CERTAIN NONLINEAR FOURTH ORDER EQUATIONS
}

\author{
W.E. TAYLOR, JR.
}

Department of Mathematics

Texas Southern University

Houston, Texas 77004

(Received August 30, 1982)

ABSTRACT. This work investigates the behavior of solutions of certain nonlinear fourth order differential equations. An example is given showing that these equations can have both oscillatory and nonoscillatory solutions simultaneously. Finally, several criteria for the existence of oscillatory solutions are established.

KEY WORDS AND PHRASES. Nonlinear fourth onder equations, oscillatory and nonoscillatory solutions, as ymptotic behavior.

1980 MATHEMATICS SUBJECT CLASSIFICATION CODE. 34 C10.

1. INTRODUCTION.

This paper is concerned with the differential equations

$$
y^{\prime \prime \prime \prime}+p(t) y^{\prime}+q(t) f(y)=0
$$

and

$$
y^{\prime \prime \prime \prime}+p(t) y^{\prime}+q(t) f(y)=r(t)
$$

where

$$
\begin{aligned}
& \text { (1) } p(t), p^{\prime}(t), q(t), r(t) \text { are continuous on }[0, \infty) \text { and satisfy } p(t)>0 \text {, } \\
& q(t)>0 \text { for all } t \geq 0 \text {. } \\
& \text { (11) f is continuous on }(-\infty, \infty) \text {, and satisfy } \frac{f(y)}{y} \geq m>0 \text { for all } y \neq 0 \text {. } \\
& \text { (111) } m(t)-p^{\prime}(t) \geq 0 \text { for all } t \geq 0 \text {. }
\end{aligned}
$$

Ne will confine ourselves to those solutions y of (1) or (2) which are defined on some half-line $\left[t_{0}, \infty\right), t_{0} \geq 0$, and are not identically zero on any sublnterval of $\left[t_{0}, \infty\right)$. Such a solution 18 termed oscillatory if 1 has a zero on every half-11ne $\left[t_{1}, \infty\right), t_{1} 2 t_{0}$ amd nonoscillatory otherwise. 
The main objective if this work is to investigate the solutions of (1.1) and (1.2) relative to their asymptotic behavior and oscillation properties. An example showing that (1.1) can have both oscillatory and nonoscillatory solutions is given after which the nonoscillatory solutions of (1.2) are examined and several oscillation criteria are derived for (1.2). The techniques used herein are similar to those used by Heidel [1] and Waltman [2] in their investigations of nonlinear third order equations, and also has points of contact with articles by this author [3] and the recent work of Lovelady [2] on nonlinear fourth order equations.

\section{MAIN RESULTS.}

Consider the functional

$$
F[y(t)]=p(t) y^{2}(t)+2 y(t) y^{\prime \prime \prime}(t)-2 y^{\prime}(t) y^{\prime \prime}(t)
$$

where $y+y(t)$ is a solution of (1.1). Computing $F^{\prime}[y(t)]$ and making the appropriate subsitutions we find that

$$
\begin{aligned}
F^{\prime}[y(t)] & =-2 y^{\prime 2}(t)-2 q(t) y(t) f(y(t))+p^{\prime}(t) y^{2}(t) \\
& \leq-2 y^{\prime \prime 2}(t)-\left(2 m q(t)-p^{\prime}(t)\right) y^{2}(t) \leq 0
\end{aligned}
$$

Thus $F[y(t)]$ is decreasing on $[0, \infty)$ since $y$ is not identically zero on any half-1ine. No solution of (2.1) can have more than one multiple zero since this would imply F could be zero at two points, contradicting the decreasing nature of $F$.

THEOREM 1. Let $y(t)$ be a solution of $(1.1)$. If $F[y(t)]>0$ on $[0, \infty)$, then

$$
\int_{a}^{\infty} y^{\prime \prime 2}(t) d t<\infty
$$

and

$$
\int_{a}^{\infty}\left(2 m q(t)-p^{\prime}(t)\right) y^{2}(t)<\infty .
$$

PROOF. Differentiating $F[y(t)]$ and integrating from a to $t$, we obtain

$$
\begin{aligned}
0<F[y(t)] & =F[y(t)]-\int_{a}^{t} 2 y^{\prime \prime 2}(s) d s-\int_{a}^{t} 2 q(s) y(s) f(y(s)) d s+\int_{a}^{t} p^{\prime}(s) y^{2}(s) d s \\
& \leq F[y(a)]-2 \int_{a}^{t} y^{\prime \prime 2}(s) d s-\int_{a}^{t}\left(2 m q(s)-p^{\prime}(s)\right) y^{2}(s) d s
\end{aligned}
$$


But this implies

$$
2 \int_{a}^{t} y^{0^{2}}(s) d s+\int_{a}^{t}\left(2 a(s)-p^{\prime}(s)\right) y^{2}(s) d s<F[y(a)]
$$

frow which the result follows.

COROLLARY. Let $y(t)$ be a solution of (1.1). If $p^{\prime}(t) \leq 0$ and $F[y(t)]>0$ for all $t \geq 0$ then

(1) $\int_{a}^{\infty} y^{\prime \prime 2}(t) d t<\infty$, (11) $\int_{a}^{\infty} q(t) y^{2}(t) d t<\infty$ and (111) $\int_{a}^{\infty}-p^{\prime}(t) y^{2}(t) d t<\infty$.

COROLLARY. Let $y(t)$ be a solution of (1.1). If $F[y(t)]>0$ and 1 Im $\operatorname{lnf}[2 \mathrm{mp}(t)-$ $\left.P^{\prime}(t)\right]>0$. then

$$
\text { (1) } \int^{\infty} y^{m^{2}}(t) d t<\infty, \text { (11) } \int^{\infty} y^{\prime 2}(t) d t<\infty \text { and }(111) \int^{\infty} y^{2}(t) d t<\infty \text {. }
$$

It should be noted that Theoren 1 and its corollaries remain equally valid if it is assuned only that $\lim _{t \rightarrow \infty} F[y(t)]>-\infty$

THBOREY 2. Suppose $\int_{0}^{\infty} p(t) d t=\infty$. If $y(t)$ is a nonoscillatory solution of $(1.1)$, $F[y(t)]>0$ on $[0, \infty)$.

PBOOF. Suppose without 1088 of generality that $y(t)>0$ on $[a, \infty)$ for some $a>0$ and assume $F[y(t)]<0$ on $[a, \infty)$. We w11l show that the latter assumption leads to a contradiction.

Consider the function

$$
H(t)=2 \frac{y^{\prime \prime}(t)}{y(t)}+\int_{a}^{t} p(s) d s
$$

Then

$$
H^{\prime}(t)=\frac{F[y(t)]}{y^{2}(t)}
$$

Thus 1.5 follows that $H(t)$ is decreasing on $[a, \infty)$ and the ratio

$$
Q(t)=\frac{y^{\prime \prime}(t)}{y(t)}
$$

mat be negative for large $t$. In fact $Q(t) \rightarrow \infty$. Thus $y^{\prime}(t)>0$ on some half-11ne [b, $\infty$ ), 
where $b \geq a$ and $y(t)$ is increasing on $[b, \infty)$.

Let $\beta<0$ be a number satisfying $Q(t)<\beta$ on $[b, \infty)$. Then

$$
y^{\prime \prime}(t)<B y(t)<\gamma<0
$$

for some $\gamma<0$. Such a $\gamma$ exists because $y(t)$ is increasing. But (2.1) implies $y^{\prime}(t) \rightarrow-\infty$ as $t \rightarrow \infty$ which is clearly impossible and the result follows.

COROLLARY. Suppose $y(t)$ is a solution of $(1.1)$ for which $F[y(t)]<0$ on some halfline $[a, \infty)$. Then $y(t)$ is oscillatory. In particular, any solution having a multiple zero is oscillatory.

EXAMPLE. Consider the equation

$$
y^{\prime \prime \prime}+\left(2+e^{-2 t}\right) y^{\prime}+y^{3}+y=0 .
$$

The function $y(t)=e^{-t}$ is a solution of (2.2). It follows from the corollary that (2.2) also has oscillatory solutions, e.g., and nontrivial solution satisfying $y(a)=y^{\prime}(a)=0$ at some $a>0$.

THEOREM 3. Suppose $y(t)$ is an oscillatory solution of (1.1) satisfying $F[y(c)]<0$ for some $c>0$. Then $y^{\prime}(t)$ is unbounded.

PROOF. Consider the function

$$
N[y(t)]=2 y(t) y^{\prime \prime}(t)=2 y^{\prime 2}(t), \quad t \geq c .
$$

An easy computation shows that $N^{\prime}[y(t)]=F[y(t)]-P(t) y^{2}(t) \leq F[y(t)] \leq F[y(c)]<0$. Thus $N[y(t)] \rightarrow-\infty$ as $t \rightarrow \infty$. The result follows by exanining $N[y(t)]$ along the zeroe of $y^{\prime \prime}(t)$ which are the extrema of $y^{\prime}(t)$.

We now turn our attention to the forced nonlinear equation

$$
y^{(4)}+p(t) y^{\prime}+q(t) f(y)=r(t)
$$

where we assume that $p, q, r$, and $f$ satisfy conditions (1) and (11) 11sted above and the additional hypothesis:

(1v)

$$
P^{\prime}(t)<0 \text { for all } t \text { on }[0, \infty) \text { and } \int_{0}^{\infty} \frac{r^{2}(t)}{P^{\prime}(t)} d t>-\infty \text {. }
$$

LEMRAA. Suppose $y$ is a solution of (2.3). Then the functional $H[y(t)]=p(t) y^{2}(t)+$ $2 y(t) y^{\prime \prime \prime}(t)-2 y^{\prime}(t) y^{\prime \prime}(t)-\int_{t}^{\infty} \frac{r^{2}(s)}{p^{\prime}(s)} d s$ is nonincreasing. 
PROOF. Taking the derivative and making the appropriate substitution for $y^{(4)}(t)$ we find that

$$
\begin{aligned}
H^{\prime}[y(t)] & =-2 q(t) y(t) f(y(t))-2 y^{\prime \prime 2}(t)+p^{\prime}(t)\left[y(t)+\frac{r(t)}{p^{\prime}(t)}\right]^{2} \\
& \leq 0
\end{aligned}
$$

Using the functional $\mathrm{H}[\mathrm{y}(\mathrm{t})]$ we can now examine the behavior of certain nonoscillatory solutions of $(2.3)$.

THEOREM 4. Suppose $y(t)$ is a nonoscillatory solution of (2.3) such that $\mathbf{H}_{\mathbf{0}}=\mathbf{H}\left[\mathbf{y}\left(\mathrm{t}_{\mathbf{0}}\right)\right]$ $<0$ for some $t_{0}$. Then

$$
\operatorname{sgn} y(t)=\operatorname{sgn} y^{\prime}(t)-\operatorname{sgn} y^{\prime \prime}(t)
$$

for all $t \geq t_{1} \geq t_{0}$.

PROOF. Let $y(t)$ be a nonoscillatory solution of $(2.3)$ such that $H\left[y\left(t_{0}\right)\right]<0$. Then it follows form the Lemma that $H[y(t)]<0$ for all $t \geq t_{0}$ and

$$
p(t) y^{2}(t)+2 y(t) y^{\prime \prime \prime}(t)-2 y^{\prime}(t) y^{\prime \prime}(t)<0,
$$

for $t \geq t_{0}$. But (2.4) implies that

$$
\frac{d}{d t}\left(\frac{y^{\prime \prime}(t)}{y(t)}\right) \leq-\frac{1}{2} p(t)<0,
$$

hence $\frac{y^{\prime \prime}(t)}{y(t)}$ is decreasing and eventually of one sign. Thus $y(t) y^{\prime \prime}(t) y^{\prime}(t) \neq 0$ for all $t$ on some half-line $\left[t_{1}, \infty\right)$. Assuming without loss of generality that $y(t)>0$ on $\left[t_{1}, \infty\right)$, the following cases must be considered:
(a) $y(t)>0, y^{\prime}(t)>0, y^{\prime \prime}(t)>0$
(b) $y(t)>0, y^{\prime}(t)>0, y^{\prime \prime}(t)<0$
(c) $y(t)>0, y^{\prime}(t)<0, y^{\prime \prime}(t)>0$
(d) $y(t)>0, y^{\prime}(t)<0, y^{\prime \prime}(t)<0$

Case (d) is clearly tmpossible. So let us suppose that (c) holds. Then

$$
p(t) y^{2}(t)+2 y(t) y^{\prime \prime \prime}(t)-2 y^{\prime}(t)<H_{0}
$$

for all $t \geq t_{1}$ and we conclude that $y(t) y^{\prime \prime \prime}(t)<\frac{1}{2} H_{0}$ on $\left[t_{1}, \infty\right)$.

Since $y(t)$ is decreasing it is easy to see that $y^{\prime \prime \prime}(t)<\frac{H_{0}}{\left.2 y_{(}\right)}$, which implies that 
$y^{\prime \prime}(t) \rightarrow-\infty$, a contradiction. So (c) is impossible. Similarly, (b) is impossible and the result follows.

Our oscillation criteria is based on theorem 4.

THEOREM 5. Suppose $\int_{0}^{\infty} q(t) d t=\infty, \lim _{t \rightarrow \infty} \sup \int_{t_{1}}^{t} r(s) d s<\infty$ and $f(y)$ is nondecreasing. Then any solution $y(t)$ of $(1.2)$ satisfying $H\left[y\left(t_{0}\right)\right]<0$, for some $t_{0}$ is oscillatory.

PROOF. Let $y(t)$ be a nonoscillatory solution of $(1.2)$ which satisfies $H\left[y\left(t_{0}\right)\right]<0$, for some $t_{0}$. Then according to our Theorem, there exists $t_{1} \geq t_{0}$ such that sgn $y(t)=$ $\operatorname{sgn} y^{\prime}(t)=\operatorname{sgn} y^{\prime \prime}(t)$ for all $t \geq t_{1}$. Assume without loss of generality that $y(t)>0$ on $\left[t_{1}, \infty\right]$. Then from $(1.2)$ we have

$$
y^{(4)}(t) \leq r(t)-f(y(t)) q(t)
$$

Integrating from $t_{1}$ to $t$ we get

$$
y^{\prime \prime \prime}(t) \leq \int_{t_{1}}^{t} r(s) d s-f\left(y\left(t_{1}\right)\right) \int_{t_{1}}^{t} q(s) d s+y^{\prime \prime \prime}\left(t_{1}\right)
$$

But based on the boundedness of $\int_{t}^{t} r(s) d s,(2.5)$ implies $y^{\prime \prime \prime}(t) \rightarrow-\infty$ as $t \rightarrow \infty$ but this would force $y(t)$ to eventually become negative, a contradiction. Hence (1.2) cannot have a nonoscillatory solution $\mathrm{y}(\mathrm{t})$ satisfying $\mathrm{H}\left[\mathrm{y}\left(\mathrm{t}_{0}\right)\right]<0$, for some $\mathrm{t}_{0}$, and the proof is complete.

THEOREM 6. Suppose $\int_{0}^{\infty} t q(t) d t=\infty$ and $\int_{0}^{\infty}|r(t)| d t<\infty$. Then any solution of (1. gatisfying $H\left[y\left(t_{0}\right)\right]<0$, for some $t_{0}$ is oscillatory.

PROOF. Suppose that $y(t)$ is a positive nonoscillatory solution of (1.2) with $H\left[y\left(t_{0}\right)\right]<0$, for some $t_{0}$. Since $\frac{f(y)}{y} \geq m$ and $y^{\prime}(t)>0$ on some half-1ine $[t, \infty) 1 t$ follows that

$$
y^{\prime \prime}(t) \leq y^{\prime \prime \prime}\left(t_{1}\right)+\int_{t_{1}}^{t} r(s) d s-m \int_{t_{1}}^{t} q(s) y(s) d s .
$$


Since $y^{\prime}(t)$ is increasing it is easily verified that

$$
y(t)>y^{\prime}\left(t_{1}\right)\left(t-t_{1}\right) \text { for all } t \geq t_{1} \text {. }
$$

Inequality (2.7) together with (2.6) shows that

$$
y^{\prime \prime \prime}(t) \leq y^{\prime \prime \prime}\left(t_{1}\right)+\left|\int_{t_{1}}^{t} r(s) d s\right|-m y^{\prime}\left(t_{1}\right) \int_{t_{1}}^{t}\left(s-t_{1}\right) q(s) d s
$$

which implies $y^{\prime \prime \prime}(t) \rightarrow-\infty$ as $t \rightarrow \infty$, a contradiction.

This completes the proof of our Theorem.

Finally we have

THEOREM 7. Suppose $\int_{0}^{\infty} p(t) d t=\infty$ and $y(t)$ is a solution of $(1.2)$ such that $H\left[y\left(t_{0}\right)\right]<0$, for some $t_{0}$, then $y(t)$ is oscillatory.

We omit the proof of Theorem 7 because of its similarity to the above proofs.

It appears from the above Theorems that any condition that Implies oscillation in (1.2) also implies oscillation in (1.1), thus it is natural to ask whether the oscillation of (1.2) implies the oscillation of (1.1). We shall leave this as an open question although the "feeling" that one gets from linear examples is that the answer is probably a negative one.

ACKNOWLEDGEMENT. This research was partially supported by Texas Southern University Faculty Research grant \#16578.

\section{REFERENCES}

1. HEIDEL, J.W. Qualitative Behayior of Solutions of a Th1rd Order Nonlinear Differential Equation, Pactfic J. Math. 27 (1968), 507-526.

2. WALTMAN, P. Oscillation Criteria for Third Order Nonlinear Differential Equations, Pac1fic J. Math. 18 ) 1966), 385-388.

3. TAYLOR, W.E., JR. Asymptotic Behavior of Solutions of Fourth Order Nonlinear Differential Equations, Proc. Amer. Math. Soc. 1 (1977), 70-72.

4. LOVELADY, D.L. An Oscillation Criterion for a Fourth Order Integrally Superlinear Differential Equation, Att1. Acad. Naz Rend. C1. Sc1. F18. Natur. 58 (1975), 532-536. 


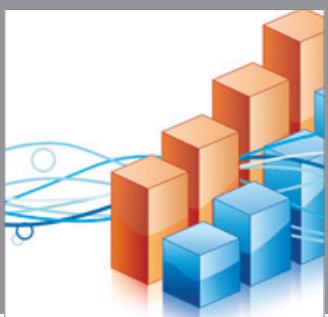

Advances in

Operations Research

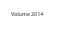

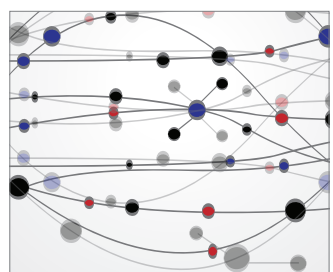

\section{The Scientific} World Journal
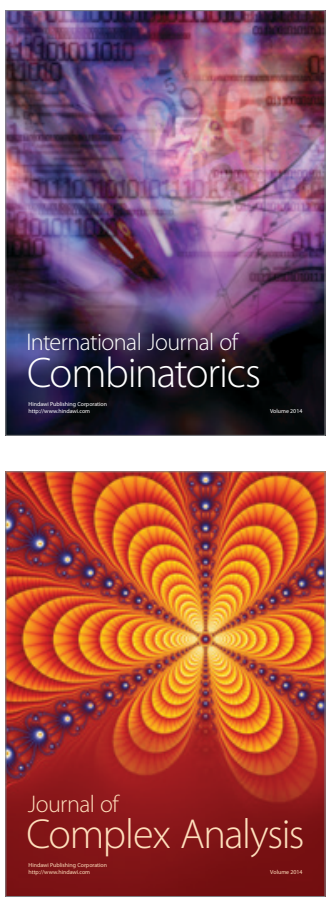

International Journal of

Mathematics and

Mathematical

Sciences
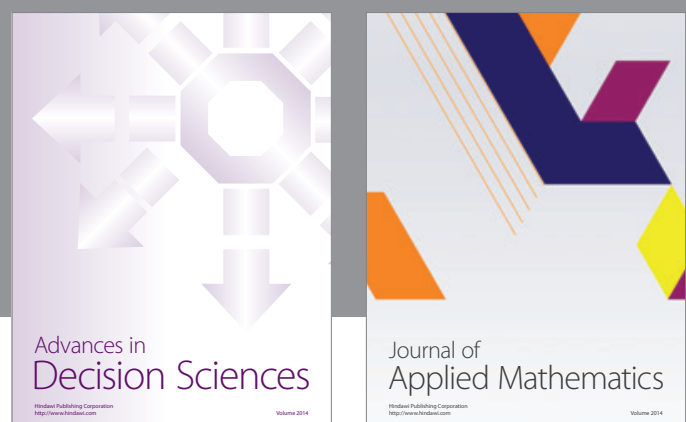

Journal of

Applied Mathematics
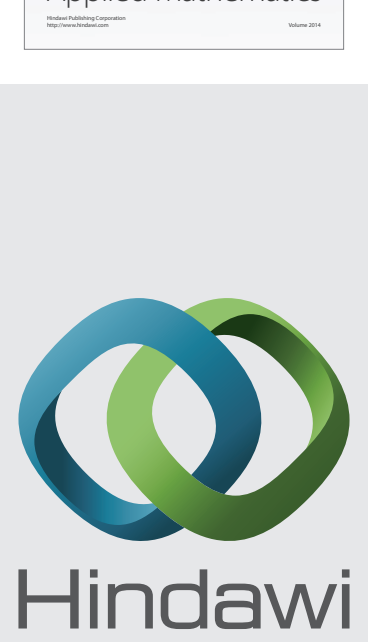

Submit your manuscripts at http://www.hindawi.com
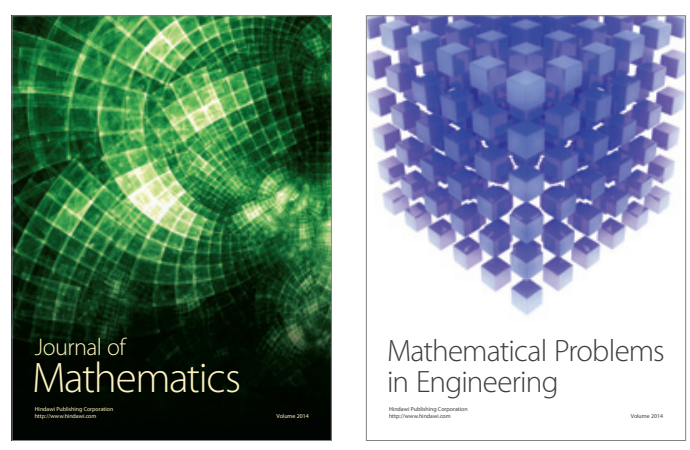

Mathematical Problems in Engineering
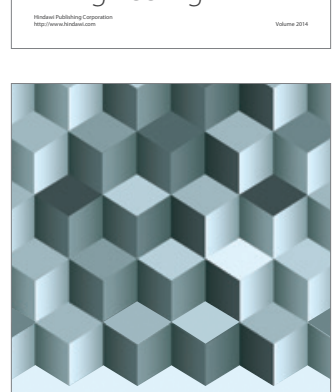

Journal of

Function Spaces
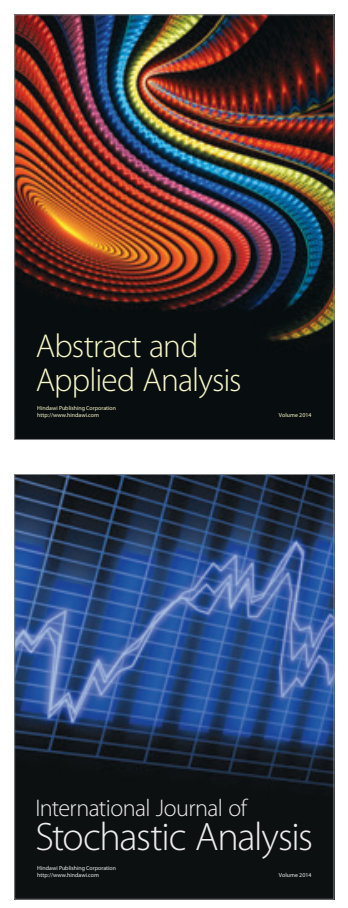

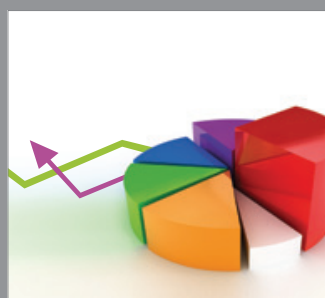

ournal of

Probability and Statistics

Promensencen
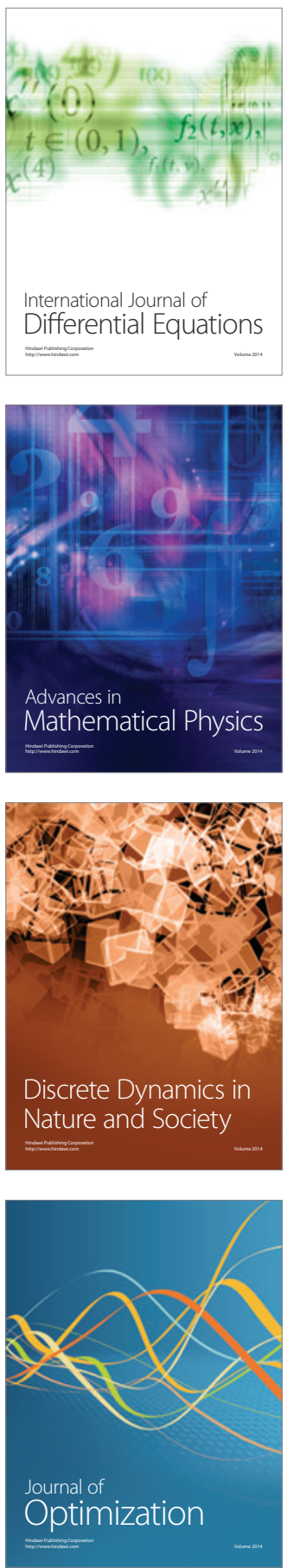